Itinéraires Itinéraires

Littérature, textes, cultures

2011-3 | 2011

Récits du corps au Maroc et au Japon

\title{
Manger et souffrir. Expériences du corps dans la littérature japonaise moderne
}

\section{Emmanuel Lozerand}

\section{(2) OpenEdition}

\section{Journals}

Édition électronique

URL : http://journals.openedition.org/itineraires/1528

DOI : $10.4000 /$ itineraires. 1528

ISSN : 2427-920X

Éditeur

Pléiade

\section{Édition imprimée}

Date de publication : 1 novembre 2011

Pagination : 103-116

ISBN : 978-2-296-55720-8

ISSN : 2100-1340

Référence électronique

Emmanuel Lozerand, « Manger et souffrir. Expériences du corps dans la littérature japonaise moderne », Itinéraires [En ligne], 2011-3 | 2011, mis en ligne le 01 novembre 2011, consulté le 19 avril 2019.

URL : http://journals.openedition.org/itineraires/1528; DOI : 10.4000/itineraires.1528

\section{(ब) $(\Theta \Theta$}

Itinéraires est mis à disposition selon les termes de la licence Creative Commons Attribution - Pas d'Utilisation Commerciale - Pas de Modification 4.0 International. 


\title{
Manger et souffrir. Expériences du corps dans la littérature japonaise moderne
}

\begin{abstract}
The modern Japanese bodies underwent violent transformations. To examine these, we shall study here the links which weaved both writer Masaoka Shiki (1867-1902) and Natsume Sôseki (1867-1916) between pain and appetite. At the first one, the tuberculosis, far from being an obstacle to the desire to eat, is in reality only sharpening the hunger. At the second on the other hand, to feed is always a distressing action which cannot engender anything but suffering. So by grasping in the root these two intense physical experiments, we hope to make resound echoes between these bodies and ours, rather than to assign them to an improbable japonity.
\end{abstract}

Keywords : Japan, body, literature, to eat, to suffer, Masaoka Shiki, Natsume Sôseki Mots clés : Japon, corps, littérature, manger, souffrir, Masaoka Shiki, Natsume Sôseki

Au tout début du $\mathrm{xx}^{\mathrm{e}}$ siècle, le 22 janvier 1901, un Japonais envoie de Londres une lettre à son épouse, demeurée à Tôkyô :

Quant aux Japonais, qu'ils se mettent en habit ou qu'ils portent la redingote, ils n'ont aucune allure. Tant que j'étais au Japon, je n'avais pas conscience de la couleur de notre peau, mais depuis que je suis ici, je suis dépité de me voir si jaune. En plus, je suis petit. Jusqu'à présent, je n'ai rencontré personne qui soit plus petit que moi. Par-dessus le marché, je ne suis pas large d'épaules. Quand j'aperçois au coin d'une rue un individu bizarre de petite taille et laid, c'est ma propre image dont une vitrine me renvoie le reflet ${ }^{1}$.

Quelques mois plus tard, le 13 juin, un de ses compatriotes écrit dans la chronique qu'il tient dans un grand quotidien :

1. «Lettre à Kyô » du 22 janvier 1901, dans Natsume Sôseki, Sôseki shokan-shû, Tôkyô, Iwanami bunko, 1990, p. 78. Trad. par Élisabeth Suetsugu dans Haltes en Mandchourie et en Corée, précédé de Textes londoniens, Paris, La Quinzaine littéraire / Louis Vuitton, 1997, p. 110. 
Sous prétexte qu'il faut améliorer la qualité des vaches japonaises, on suggère que leur lait est mauvais, mais c'est absolument faux, c'est simplement que, comme elles n'en donnent pas une grande quantité, elles ne sont pas rentables économiquement. Et ce n'est pas non plus que leur viande serait mauvaise, car non seulement c'est absolument faux, mais en outre le bœuf de Kôbe est considéré comme l'une des viandes les plus savoureuses du monde. Alors pourquoi faudrait-il améliorer la qualité de nos vaches? Tout simplement parce qu'elles ne donnent pas assez de chair par rapport à ce qu'elles mangent. Autrement dit, parce qu'elles ne sont pas rentables économiquement. [...]

Au Japon, ne serait-ce que parce qu'il s'agit d'un pays insulaire, on ne produit que de petites choses, mais, en revanche, on y trouve des saveurs intenses et concentrées. [...] Cependant dans un monde unifié comme celui d'aujourd'hui, ce qui n'est pas rentable économiquement ne peut l'emporter dans la lutte pour la vie [...].

À ce propos, il me revient que l'an passé on a fait grand cas du problème de l'amélioration de la race humaine. Si l'on peut améliorer les humains comme on améliore les bœufs, d'ici quelques années les Japonais auront de grands corps qui ne le céderont en rien à ceux des Occidentaux, on obtiendra ainsi une race forte, qui ignorera la maladie, $[\ldots]$ une race rentable économiquement. Mais alors la saveur singulière à la nature innée des Japonais subsistera-t-elle encore? Je n'en suis pas certain ${ }^{2}$.

Ces deux hommes sont nés trente-quatre ans plus tôt, à la veille de la Restauration impériale de Meiji. Le premier d'entre eux, arrivé à Londres l'année précédente, est un chercheur en langue et littératures anglaises. Le second est un journaliste qui vit à Tôkyô, où il est responsable d'une rubrique de poésie dans un grand quotidien. Le premier souffre des nerfs. On le dit dépressif, voire dément. Le second est tuberculeux, une tuberculose qui carie ses os et le force depuis six années à demeurer presque immobile sur sa couche.

Par ces deux citations liminaires, je souhaiterais rappeler que les corps japonais ont connu dans la seconde moitié du XIX ${ }^{e}$ siècle de profondes transformations. Celles-ci ne sont certes pas les premières de leur histoire, et il ne faudrait surtout pas laisser accroire qu'un corps « traditionnel » aurait alors laissé la place à un corps « moderne ». Les corps avaient déjà beaucoup changé au Japon avec l'urbanisation massive des XVII et XVIII ${ }^{e}$ siècles, grâce entre autres à la « domestication » $\left(\right.$ taming $\left.^{3}\right)$ des corps guerriers médiévaux, qui avaient succédé aux corps aristocratiques de l'époque du Dit du Genji, eux-même bien différents des corps archaïques dont témoigne indirectement

2. Masaoka Shiki, Bokujû itteki, Tôkyô, Iwanami bunko, 1999, p. 142-144. Sauf avis contraire, les traductions sont originales.

3. Voir Ikegami Eiko, The Taming of the Samurai. Honorific Individualism and the Making of Modern Japan, Cambridge Massachusetts / London, Harvard University Press, 1995. Il serait instructif de tester méthodiquement sur l'exemple japonais la validité des hypothèses de Norbert Elias sur le « processus de modernisation », tout comme de celles de Max Weber sur la « rationalisation». 
la Chronique des choses anciennes (Kojiki) du VIII' siècle - corps qu'il faut d'ailleurs se garder d'assimiler hâtivement à ceux dont l'archéologie date les premières traces sur l'archipel au Paléolithique, il y a quelque 45000 ans $^{4}$.

Mais les transformations du XIX ${ }^{e}$ siècle furent malgré tout brutales et lourdes de conséquences, en particulier parce que le contact avec l'Occident entraîna une confrontation, une concurrence, avec des corps autres ${ }^{5}$. Que les Japonais aient alors été perçus avec mépris comme des « singes $^{6}$ », placés bien bas sur l'échelle des races en vigueur en Occident, ou comme des «poupées » séduisantes, relève d'une vision orientaliste qu'Albert Memmi a résumé de manière limpide : « $\mathrm{Ah}$, ils ne sont pas beaux, le corps et le visage du colonisé ${ }^{7} !$ » Mais cette rencontre avec les corps et les regards de $1^{\prime} O c c i d e n t^{8} n$ 'est pas le seul élément à prendre en compte. La période se caractérise aussi par l'introduction de nouvelles pratiques, de nouvelles références, et en particulier par une action accrue de l'État, qui cerne désormais de plus près le corps des citoyens ${ }^{9}$, et par une emprise nouvelle de la technique et des machines, comme le train et l'électricité ${ }^{10}$.

On ne peut donner ici qu'un aperçu très sommaire de la multitude de changements qui affectèrent alors les pratiques corporelles au Japon, que ce soit dans la santé, où l'hygiène publique supplanta les pratiques anciennes de «nutrition du principe vital », dans les mœurs, où nourriture, habitat, vêtements, coiffure furent bouleversés, dans le monde du travail, la conduite de la guerre ${ }^{11}$, l'éducation, les loisirs, la sexualité ou la vie familiale. Les

4. Pour un regard sur la préhistoire japonaise, voir les travaux de Laurent Nespoulous, dans Jean-Paul Demoule et Pierre Souyri (dir.), Archéologie et patrimoine au Japon, Paris, Maison des sciences de l'homme, 2008; ou le bilan de Pierre Souyri, Nouvelle histoire $d u$ Japon, Paris, Perrin, 2010.

5. Est-il besoin de rappeler qu'il n'y a pas de peuple japonais « autochtone »? Que des vagues de populations se sont croisées sur l'archipel?

6. «Les Japonais sont des singes » : dans Mon cœur mis à nu, Baudelaire se fait l'écho d'une réaction de l'opinion à la visite en France de la première ambassade japonaise en 1864.

7. Albert Memmi, Portrait du colonisé, Paris, Gallimard, coll. «Folio », 2002, p. 135.

8. Voir notre article, «Le papillon, la cloche et la poupée. Résonances politiques d'une chanson populaire », Cipango. Cahiers d'études japonaises, n 12, 2005, p. 172-173.

9. Pour la France, voir Michel Foucault, Sécurité, territoire, population, Paris, Gallimard/ Seuil, 2004; et Gérard Jorland, Une société à soigner. Hygiène et salubrité publique en France au XIXe siècle, Paris, Gallimard, 2010.

10. Voir à ce sujet la thèse de Philippe Cominetti, L'Inspiration scientifique dans le roman post-naturaliste. Du naturalisme français à la littérature japonaise du premier $X X^{e}$ siècle, sous la dir. d'Alain Rocher, Université Bordeaux 3, 2005.

11. Natsume Sôseki se fait l'écho de la violence inédite de la guerre russo-japonaise dans Le Goût en héritage (Shumi no iden, 1906), trad. par Hélène Morita dans Échos illusoires du luth, Paris, Le Serpent à plumes, 2008. Voir aussi Dany Savelli (dir.), Les Carnets de l'exotisme, $\mathrm{n}^{\circ} 5$, Faits et imaginaires de la guerre russo-japonaise, 2005, et en particulier l'article de Tsukamoto Toshiaki, "Natsume Sôseki et la guerre russo-japonaise », p. 341-358. 
rythmes de vie furent modifiés de fond en comble ${ }^{12}$, l'espace transformé au gré de profondes restructurations urbaines et de l'extension des réseaux de transport. Le rapport au passé se métamorphosa puisque fut alors « inventé » un corps « traditionnel ${ }^{13}$ ». Les représentations changèrent profondément, marquées elles-mêmes par des évolutions techniques et artistiques, que ce soit en peinture, en photographie ou dans le cinéma.

Étudier l'ensemble de ces transformations serait ouvrir un gigantesque chantier, dans lequel il faudrait tenir compte d'innombrables variations et nuances, en fonction des différences de régions, de classes sociales, de genre, de générations... On voudrait ici plus modestement tenter d'approcher un tant soit peu quelques-uns de ces corps en mouvement, décomposés et recomposés par l'histoire. Et la littérature peut ici apporter une contribution irremplaçable parce que, loin des abstractions parfois trop distantes des sciences sociales, elle donne accès à des expériences singulières.

Les deux écrivains retenus pour cette étude, auteurs des deux textes cités, sont nés l'un et l'autre en 1867, l'un à Edo (sur le point de devenir Tôkyô), l'autre à Matsuyama dans l'île de Shikoku. Ils se rencontrèrent en classe préparatoire à l'examen d'entrée à l'Université de Tôkyô, en 1889, et devinrent aussitôt de très proches amis ${ }^{14}$. Et pourtant que de différences opposent Natsume Sôseki (1867-1916), unanimement considéré comme le grand romancier japonais du Xx ${ }^{\mathrm{e}}$ siècle, et Masaoka Shiki (1867-1902), respecté comme celui qui a donné une nouvelle vie au haiku! Dès leur jeunesse estudiantine, leurs tempéraments s'opposent. À Shiki qui ne pense qu'à écrire, écrire, écrire, Sôseki répond qu'il faut d'abord lire, lire, réfléchir et déterminer des idées justes. Si je les ai réunis ici, c'est précisément parce que l'un et l'autre témoignent de deux dimensions essentielles du corps, l'appétit et la douleur ${ }^{15}$, mais en les nouant de manière radicalement contrastée. Malgré la maladie qui le condamne à l'invalidité, l'immobilité et la souffrance, Shiki est un homme de désir, insatiable, alors que Sôseki, affaibli par la dépression et les ulcères de l'estomac, est comme frappé d'aboulie. En se mettant à leur

12. Sur la « naissance du retard » dans le Japon moderne, voir Hashimoto Takehiko et Kuriyama Shigehisa, Chikoku no tanjô, Tôkyô, Sangensha, 2001.

13. Une mode du bushidô sévit par exemple dans les années 1890 et 1900.

14. Voir Jean-Jacques Origas, «Une amitié : Shiki le poète et Sôseki le prosateur », dans La Lampe d'Akutagawa. Essais sur la littérature japonaise moderne, Paris, Les Belles Lettres, 2008, p. 149-169.

15. Sur les liens entre littérature et nourriture dans la littérature moderne, voir Tomoko Aoyama, Reading Food in Modern Japanese Literature, Hawai'i, University of Hawai'i Press, 2008, et, de manière plus anecdotique, Tsubouchi Toshinori, Shiki no kokoa Sôseki no kasutera, Tôkyô, Nihon hôsô shuppan kyôkai, 2006. Sur les liens entre maladie et littérature, voir Karatani Kôjin, Nihon kindai bungaku no kigen [1970], Tôkyô, Kôdansha bungei bunko, 1988, et en particulier le chapitre IV : «Yamai to iu imi », p. 129-152. Il existe une traduction anglaise de cet essai par Brett De Bary, sous le titre Origins of Modern Japanese Literature, Durham, Duke University Press, 1993. 
écoute, dans des expériences humaines premières, on espère ainsi donner à rencontrer deux corps japonais « vrais », aux antipodes de toute imagerie martiale (des samouraïs aux robots) ou érotique (des geishas aux lolitas).

\section{Souffrir, mais manger : Masaoka Shiki}

Le 16 mars 1901, dans la chronique qu'il tient dans le journal Nihon, Shiki cite une lettre envoyée par un admirateur qu'il n'a jamais rencontré :

C'était la première fois que je voyais une photo de maître Shiki et j'ai été très surpris. D'avoir ainsi accompli ce grand travail tout en combattant depuis tant d'années contre le démon de la maladie exige un courage étonnant, qui transparaît entre ces sourcils, mais avec son aspect décharné je dirais volontiers qu'il ressemble à un arhant vivant ${ }^{16}$.

Les « arhant » (rakan), saints de la plus vieille tradition bouddhique, étaient des pratiquants rigoureux des défenses. Ils se présentent donc généralement sous la forme de statues décharnées. Mais quel que soit son état physique, l'appétit de Shiki est intact. Il publie parfois comme des pages volées à un journal de bord qu'il ne tient pas régulièrement. On y mesure la place des repas dans sa vie quotidienne, le bonheur que ceux-ci lui apportent :

-29 juillet mardi. Temps couvert.

Vers neuf heures, après être passé à la selle, je souffre, et comme d'habitude je prends des anesthésiques. Avant même que les médicaments n'aient fait leur effet, je me sens déjà joyeux. [...]

Un bol de lait et un peu de pain. [...]

Pour le déjeuner, des sushis à la lie de tôfu. Il s'agit d'un mélange de chair de poisson avec des résidus de caillé de soja laxatif.

Nouvelle sieste. À mon réveil, j'avale de la soupe sucrée de haricots rouges en poudre. [...]

Dîner. Trois bols de riz, grillades, patates douces, aubergines, pois, légumes confits aux trois condiments. Tout me paraît délicieux ${ }^{17}$.

Dans les pires moments, manger est pour lui le signe et le moyen même du retour à la vie. Le 15 mai 1902, par exemple, alors qu'il est au plus mal, il compose ce qu'il croit être son dernier verset :

Par ce geste, je voulais dire que, si je m'endormais ainsi, ce serait mon poème d'adieu, mais, comble du ridicule, l'après-midi j'oubliai peu à peu ma douleur et, tout réjoui de me souvenir que c'était aujourd'hui la fête à Negishi, je changeai complètement d'état d'âme, je me régalai de caillé de soja et bu une coupe de saké pour célébrer l'événement : ces derniers temps, je me suis complètement fourvoyé, mais finalement cela se termine bien; comme il reste encore quinze jours en mai, je me demande comment je vais bien pouvoir les occuper.

16. Masaoka Shiki, Bokujû itteki, op. cit., p. 62-63.

17. Masaoka Shiki, Byôshô rokushaku, Tôkyô, Iwanami bunko, 2000, p. 131-132. 
- Le 15 mai, c'était la fête au sanctuaire Mishima de Kami Negishi et ce jour-là, comme chaque année, il s'est mis à pleuvoir. Cependant avec un bouillon au caillé de soja, de jeunes pousses en sauce, le tout accompagné d'un verre de vin, ce fut un plaisir sans mélange :

Pousses de bambous

Et bourgeons en sauce

C'est la fête ${ }^{18}$ !

De manière plus fondamentale encore, manger, découvrir avec bonheur des plats nouveaux, est un moyen de prolonger la vie, comme il l'explique avec humour en relatant son festin du 28 février 1901 :

Aujourd'hui est prévu un festin de grande cuisine dans le style de la cérémonie du thé. [...]

Vers les cinq heures, on sert le repas. Fumoto joue le rôle du maître de maison. Voici le menu :

- La soupe est un miso de Mikawa filtré, aux chrysanthèmes sauvages. J'en reprends deux fois.

- Le plat vinaigré est une carpe au vinaigre doux. Il paraît que c'est un assaisonnement secret. Je mange tout, sauf le raifort râpé, mais prenant conscience de la règle qui veut que dans ce genre de cuisine on aille jusqu'au bout des plats, sans rien laisser, je commence soudain à hésiter, et je mélange le raifort dans la soupe pour l'absorber. Éclat de rire général.

[...] Je mange à volonté riz et soupe, mais pour le saké, je dois me limiter, et quand on en sert avec les daurades, j'en bois à petites gorgées, entre deux bouchées de riz. [...] Le repas terminé, on verse de l'eau salée dans un pot et on nous en sert. Comme c'est mon premier festin dans le style de la cérémonie du thé, ma vie va être prolongée de soixante-dix jours, et pour m'en souvenir je le consigne par écrit ${ }^{19}$.

Cet appétit inépuisable ne disparaît pas, alors même que les effets de la maladie se font de plus de plus en plus rudes, provoquant d'atroces souffrances dentaires et intestinales. Shiki écrit ainsi le 15 mars 1901 :

Plaisir de la promenade, du voyage, plaisir d'assister à du nô ou à du kabuki, plaisir d'aller écouter les conteurs, de regarder attractions et spectacles forains, plaisir d'aller voir une exposition, plaisir d'observer les cerisiers, la lune ou la neige, plaisir d'aller prendre des bains chauds avec son épouse, plaisir des quartiers réservés et de l'alcool, de poser sa tête sur les genoux d'une beauté, plaisir d'organiser une session de haiku dans un pavillon de thé de Meguro et de se frapper la panse pleine de riz aux châtaignes, plaisir de contempler les vastes étendues de Musashino depuis le mont Dôkan-yama, et de mordiller des kakis dans un salon de thé de Gakehana. Liberté de marcher, liberté de s'asseoir et de s'étendre, liberté de se retourner sur sa couche, de déplier ses jambes, liberté de rendre des visites, de participer à une réunion, liberté d'aller aux cabinets, liberté de chercher des livres, liberté de sortir se calmer quand on s'est mis en colère, liberté de foncer au dehors quand un

18. Ibid., p. 20-21.

19. Masaoka Shiki, Bokujû itteki, op. cit., p. 43-45. 
incendie ou un tremblement de terre menacent. Tous les plaisirs, toutes les libertés m'ont été arrachés et seuls demeurent, fragiles, un unique plaisir et une unique liberté : le plaisir de boire et de manger, la liberté d'écrire. Mais à présent les douleurs locales sont violentes et la liberté d'écrire m'a presque entièrement quitté; estomac et intestins ont fini par s'affaiblir et je me vois privé de la plus grande part de mon plaisir de manger. Ah! quel plaisir peut-il bien me rester dans les jours et les mois à venir?

[...] Ce que je souhaite, c'est que Dieu commence par me donner un jour de répit, que je puisse pendant vingt-quatre heures mouvoir mon corps à mon aise, puis qu'il me redonne l'envie de manger de tout. Alors, après cela, peut-être penserai-je paisiblement au bonheur éternel ${ }^{20}$ ?

Envers et contre tout, Masaoka Shiki est donc un homme d'appétence. Bien qu'il soit frappé par une terrible maladie, qui ne cesse de gagner du terrain, limite férocement ses possibilité de vivre, et de manger, son avidité gourmande n'est jamais altérée.

Adhérant sans réticence au mouvement de son époque, il s'est lancé de toute son énergie dans des combats pour la réforme de la littérature. Il croit dans l'affirmation nationale de son « petit pays » menacé, dont il veut développer et magnifier les ressources, malgré les inquiétudes ${ }^{21}$. Mais sa perception d'un danger d'acculturation n'implique ni repli ni fermeture. Shiki n'est pas un réactionnaire, ni un nostalgique. Si enraciné qu'il soit dans le bonheur de son terroir de Shikoku ${ }^{22}$, il sait échapper à la bêtise d'un localisme étroit. Fanatique de base-ball, il aimait le lait, le chocolat chaud et les mandarines américaines.

De manière paradoxale et tragique, Masaoka Shiki a tellement épousé cette dynamique de modernisation qu'il en a attrapé la maladie par excellence, la tuberculose ${ }^{23}$. Mais il n'y a chez lui aucun pathos, aucun lamento, même s'il ne réfrène pas les cris que de violentes douleurs l'amènent à pousser. Il ne relate pas non plus quelque combat héroïque contre le mal, ne fait pas de ses souffrances le point de départ ou le prétexte d'une quelconque introspection, d'une élévation spirituelle ou d'une métaphorisation. La maladie chez Shiki est une donnée. Elle détermine les possibilités de son corps diminué, mais n'altère en rien sa vitalité. Peut-être est-ce là

20. Ibid., p. 61-62.

21. Sur les accomplissements de Shiki, voir Jean-Jacques Origas, « Masaoka Shiki », dans La Lampe d'Akutagawa, op. cit., p. 145-148.

22. Sur l'attachement de Shiki à son pays natal, voir Jean-Jacques Origas, « Ce qui est loin, ce qui est proche », dans La Lampe d'Akutagawa, op. cit., p. 191-205.

23. Voir Fukuda Mahito, Kekkaku no bunkashi, Tôkyô, Nagoya daigaku shuppankai, 1995, et Kekkaku to iu bunka, Tôkyô, Chûo kôronsha, 2001. Voir aussi Kaneko Mitsuharu, Zetsubô no seishinshi, Tôkyô, Kôdansha bungei bunko, 1996, p. 66-67 (trad. par Benoît Grévin, sous le titre Histoire spirituelle du désespoir, Paris, Éditions Ens rue d’Ulm, 2009, p. 57-59). Sur le choléra, voir Philippe Chemouilli, «Les épidémies de choléra et la mise en place d'un système d'hygiène moderne », dans Cipango. Cahiers d'études japonaises, nº 11, 2004, p. 173-208. 
une forme particulière de courage ${ }^{24}$ ? Ses textes sont en tout cas traversés d'une étonnante allégresse, d'une constante jubilation ${ }^{25}$.

\section{Manger et souffrir : Natsume Sôseki}

Natsume Sôseki a bâti son œuvre de fiction en quelques années, de 1905 à 1916, en une dizaine de romans ${ }^{26}$ où se déploie un imaginaire cohérent. Dans ces récits, l'appétit des personnages est comme attaqué à la racine. Il ne leur reste plus, souvent, qu'une simple faim physiologique, accompagnée d'un sentiment de dégoût et de malaise.

C'est peut-être dans Le Mineur (Kôfu) en 1906, que Sôseki a peint de la manière la plus cru(ell)e la décomposition de l'humanité d'un personnage. Un jeune homme marche dans la nuit. Il fuit une famille bourgeoise, la capitale. Tombé entre les mains d'une sorte de maquignon, qui recrute des bras pour la mine, il part à sa suite dans une quasi-descente aux enfers. La première station de celle-ci a lieu dans une infâme gargote où il tombe en arrêt, affamé, devant des « beignets ronds »:

Je fixai intensément le plat, il était noir de mouches. [...] Sur les croûtes jaunes, huileuses, elles formaient toutes sortes de boursouflures noires, désordonnées.

Je mordis dedans. Ma langue éprouva une sensation huileuse et, tout de suite après, mes papilles gustatives furent attaquées par la pâte de haricots rance. [...]

Une chose a beau vous sembler sale au point que vous hésitiez à y goûter, une fois lancé, vous êtes capable de la manger sans que vos nerfs en soient tellement irrités. [...] À cette époque pourtant, je m'ébahis de constater que ces beignets-là, non seulement je les mangeais, mais que j'avais envie d'en manger davantage. J'avais faim, voilà ${ }^{27}$.

La chute se poursuit lors du « repas » suivant, composé de « patates douces» :

Les patates étaient maigrichonnes, humides, rouges et noires, la peau crevée par endroits, et la chair qui s'en échappait comme patinée de vert de gris ${ }^{28}$.

Une étape ultime est atteinte à la mine, dans la baraque longue où le personnage finit par échouer :

Mon estomac était totalement vide. [...] Saisissant les baguettes, je les plongeai dans le bol pour enfin... Que se passait-il? Je fus médusé. Le riz

24. Voir à ce sujet, Masaoka Shiki, Bokujû itteki, op. cit., p. 85-86.

25. Voir Emmanuel Lozerand, «Le canari de Shigeeko. Masaoka Shiki, le réel, l'écriture », dans Japon Pluriel 6. Actes du sixième colloque de la Société française des études japonaises, Arles, Picquier, 2006, p. 127-139.

26. Pour une présentation de Sôseki, voir Jean-Jacques Origas, « Natsume Sôseki », dans La Lampe d'Akutagawa, op. cit., p. 93-97.

27. Natsume Sôseki, Natsume Sôseki zenshû, vol. 4, Tôkyô, Chikuma bunko, 2004, p. 441-443. Trad. par Hélène Morita, Le Mineur, Paris, Le Serpent à plumes, 2000, p. 21-23. 28. Ibid., p. 490; Le Mineur, op. cit., p. 78-79. 


\begin{abstract}
glissait le long des baguettes, impossible de l'accrocher. Je recommençai en imprimant aux baguettes plus d'énergie et en les enfonçant jusqu'au fond : même résultat, le riz dégoulinait sur le bois, il ne se décidait pas à quitter le bol. [...] Et j'introduisis enfin dans ma bouche un peu de ce riz récalcitrant. Le goût incroyable que je sentis alors fit que mon âme tout entière se réfugia sur le bout de ma langue, et c'était comme si j'oubliais les rires méchants, les mineurs eux-mêmes et ma faim. Inconcevable de parler de riz à propos de ça. C'était plutôt une terre sableuse. Décrire cette matière inconsistante mélangée à ma salive et la sensation que j'en éprouvai alors est tout à fait hors de ma portée ${ }^{29}$.
\end{abstract}

Selon une logique similaire, le personnage est également atteint dans son sommeil, qu'il va pourtant trouver dans des conditions toujours plus atroces. On aboutit ainsi en quelques dizaines de pages, qui ne racontent que quelques jours d'une vie, à une véritable dissolution de l'individualité d'un être ordinaire, à une forme de déshumanisation d'un corps soumis à une logique économique et industrielle brutale, qui le réduit à une forme d'animalité ${ }^{30}$.

Cette atteinte portée à l'appétit, au désir et au plaisir de manger, se retrouve de manière éparse dans l'ensemble de l'œuvre de Sôseki où l'acte de se nourrir est réduit à un instinct élémentaire, nécessaire, mais déplaisant, en tout cas problématique, qui ne cesse d'engendrer soucis et difficultés. C'est le cas par exemple dans Botchan (1906), où une des seules distractions du héros, aller manger au restaurant, est gâchée par la bêtise provinciale. Sans cesse espionné dans les moindres de ses faits et gestes, il est systématiquement confronté à un écho de ses repas de la veille sur le tableau noir des salles de classe où il enseigne : «Professeur nouille et friture », « Succulentes les boulettes de bordels ${ }^{31} »$.

De même, dans le célèbre Je suis un chat (Wagahai wa neko de aru, 1905), les deux personnages principaux, Kushami, le maître de maison, ainsi que le « chat »-narrateur, ne peuvent avoir un rapport simple à la nourriture. Kushami souffre en effet de difficultés gastriques qu'il consigne dans son journal intime :

L'autre jour, on m'a dit que l'estomac va mieux quand on cesse de déjeuner le matin. J'ai essayé deux ou trois jours, mais le seul effet a été des borborygmes. Une autre personne m'a conseillé d'abandonner les légumes confits au sel et au vinaigre. D'après elle, ils sont la cause de tous les troubles de l'estomac. [...] Selon un troisième encore, rien ne vaut les massages abdominaux, mais pas de façon ordinaire. [...] A. m'a recommandé de ne rien manger de solide. Je n'ai bu que du lait pendant tout un jour, mais mon ventre faisait des glouglous

29. Ibid., p. 553-554; Le Mineur, op. cit., p. 157-158.

30. Voilà comment sont décrits les visages des mineurs : «J'aurais eu beau chercher, je n'aurais pu découvrir la moindre rondeur, la moindre chaleur, la moindre douceur sur ces visages. Bref, c'étaient des visages d'une absolue brutalité. » (Ibid., p. 544; Le Mineur, op. cit., p. 146).

31. Natsume Sôseki, Botchan, Tôkyô, Iwanami bunko, 1989, p. 46-48. Trad. par Hélène Morita, Botchan, Paris, Le Serpent à plumes, 1993, p. 47-50. 
qui évoquaient une inondation et j'ai passé une nuit blanche. B. m'a suggéré de respirer avec le diaphragme afin de donner de l'exercice à mes intestins; de cette façon les mouvements de l'estomac se régulariseraient tout naturellement. [...] C. m'a recommandé de manger des nouilles de sarrasin; je me suis aussitôt mis aux pâtes arrosées de sauce de soja et aux vermicelles tour à tour, mais le seul résultat que j'en ai retiré a été la diarrhée. J'ai essayé sans aucun succès tous les moyens bons à guérir cette vieille maladie de l'estomac ${ }^{32}$.

Le « chat » qui se moquait de son maître va pourtant lui aussi se retrouver piégé par la nourriture quelques pages plus loin, quand il décide de s'attaquer à un gâteau de riz gluant :

Enfin, faisant porter tout mon poids sur le bol comme pour y tomber, je plante mes dents fermement dans un petit coin du morceau de mochi. Avec la vigueur que j'ai mise à mordre, $\mathrm{j}$ 'aurais dû pouvoir couper presque n'importe quoi, mais à ma stupéfaction, mes dents restent immobilisées quand je tente de retirer ma prise! J'essaie de mordre plus profondément, mais je ne peux plus remuer les mâchoires. Je me rends alors compte que les mochi sont des démons, mais il est déjà trop tard. Comme un prisonnier dans un marécage se débat pour en sortir, plus je mords, plus ma bouche devient lourde, et plus mes dents sont progressivement enserrées. Elles ont bien prise sur le mochi, mais celui-ci ne cède pas et je ne peux plus rien faire. [...] Au comble du martyre, je secoue ma queue en tous sens, je dresse et couche mes oreilles, mais tout en vain; d'ailleurs, ma queue et mes oreilles n'ont aucun rapport avec le mochi et je renonce quand je m'aperçois que je les agite en pure perte; à la longue, je conclus que la seule chose à tenter est de repousser le mochi avec mes pattes de devant, et je donne d'abord quelques coups de ma patte droite près de ma bouche, mais le piège qui me retient ne se relâche pas pour si peu, je presse alors ma patte gauche et je décris des cercles furieux avec ma tête, en prenant ma bouche comme centre, mais cette danse ne suffit pas à conjurer le démon. Puis je me dis que la patience s'impose et j'appuie alternativement à gauche et à droite; mes dents restent toujours collées dans le mochi. «Ah, ça suffit! » m'emporté-je, et j'utilise mes deux pattes ensemble. À mon grand étonnement, je réussis à me tenir sur mes pattes de derrière, avec la vague impression de ne plus être un chat. Mais cela n'a aucune importance dans ma situation et, prenant la résolution de lutter jusqu'à ce que ce diable de mochi lâche prise, je me racle le visage dans tous les sens. L'agitation furieuse de mes pattes de devant me fait parfois perdre l'équilibre, que je dois rattrapper avec mes pattes de derrière, et je ne peux pas rester sur place; je parcours ainsi toute la cuisine en bonds désordonnés ${ }^{33}$.

Toute dimension comique disparaît d'À l'équinoxe et au-delà (Higan sugi made, 1912) où l'acte de manger est directement associé à la mort subite d'une jeune enfant de deux ans qui s'écroule soudain, au milieu de

32. Natsume Sôseki, Wagahai wa neko de aru, Tôkyô, Shinchô bunko, 1974, p. 30-31. Trad. par Jean Cholley, Je suis un chat, Paris, Gallimard, 1978, p. 47-48.

33. Ibid., p. 33-35; Je suis un chat, op. cit., p. 50-52. 
son repas ${ }^{34}$. Cette horreur se retrouve à nouveau dans Clair-obscur (Meian, 1916), le dernier roman de Sôseki, inachevé, dont l'incipit est particulièrement audacieux :

Après l'avoir examiné avec une sonde, le médecin fit descendre Tsuda de la table d'opération :

« Comme je l'imaginais, ça va jusqu'à l'intestin. La dernière fois que je vous ai examiné, j'ai remarqué, au milieu, un bourrelet de cicatrices et j'ai hâtivement conclu que c'était l'extrémité. C'est pourquoi je vous ai parlé comme je l'ai fait. Aujourd'hui, pour dégager le passage, j'ai gratté cette grosseur et il y en avait encore plus loin ${ }^{35}$. "

Tsuda quitte ensuite le cabinet médical :

Il était déprimé lorsqu'il prit le train. Au milieu d'une foule si compacte qu'il pouvait à peine bouger, il s'abandonnait à ses réflexions sur lui-même, tout en se retenant à une poignée. La douleur lancinante de l'année précédente montait à nouveau sur la scène de sa mémoire. Il revoyait clairement sa piteuse figure allongée sur le lit blanc. Il entendait nettement son propre gémissement, pareil au grognement d'un chien qui ne parvient pas à se libérer de ses chaînes pour fuir. Ensuite, les reflets des lames glacées, le cliquètement de leurs tranchants qui se heurtent, et enfin, soudain, une force de pression effrayante expulsait, d'un seul coup, l'air hors de ses poumons, puis une violente souffrance qui n'était due qu'à l'impossibilité pour cette masse d'air compressée de se contracter : toutes ses impressions assaillirent sa mémoire ${ }^{36}$.

Ces scènes douloureuses trouvent un écho direct dans les textes autobiographiques que l'écrivain a consacré à sa maladie, en particulier Choses dont je me souviens (Omoidasu koto nado, 1910) :

Une vieille femme venait trois fois par jour prendre commande de mes repas, mais j'avais beau lui demander quelque mets susceptible de me plaire, dès que mon regard se tournait vers les assiettes disposées sur la petite table, sans que je sache d'où cela venait, je me sentais envahi de dégoût pour toute cette nourriture et n'arrivais même pas à toucher aux baguettes. Puis j'ai eu un haut le cœur.

D'abord je vomis en abondance un liquide jaunâtre qui ressemblait à une décoction ${ }^{37}$.

La situation s'aggrave et les douleurs abdominales deviennent intolérables :

Sans cesse, depuis l'aube jusqu'à la tombée du jour, j'avais envie d'arracher au plus vite cette partie de mon corps et de la lancer aux chiens. [...]

34. Natsume Sôseki, Higan-sugi made, Tôkyô, Iwanami bunko, 2004, p. 179-180. Trad. par Hélène Morita, À l'équinoxe et au-delà, Paris, Le Serpent à plumes, 1995, p. 232-233. 35. Natsume Sôseki, Meian, Tôkyô, Iwanami bunko, 2005, p. 7. Trad. par René de Ceccatty et Nakamura Ryôji, Clair-obscur, Paris, Rivages, 1993, p. 9.

36. Ibid., p. 9; Clair-obscur, op. cit., p. 11.

37. Natsume Sôseki, Omoidasu koto nado, Tôkyô, Iwanami bunko, 2004, p. 32; trad. par Élisabeth Suetsugu, Choses dont je me souviens, Arles, Picquier, 2000, p. 49. 
C'était comme si l'on fouaillait à l'intérieur de ma poitrine avec un bâton pour mélanger tout ce qui s'y trouvait, ou encore comme si mes entrailles, cherchant à remonter à la surface, formaient à grand fracas des vagues monstrueuses et désordonnées ${ }^{38}$.

Cette destruction de l'appétit si caractéristique de l'univers de Sôseki n'est pas qu'une simple donnée contingente. L'écrivain établit en effet un lien de cause à effet entre ses soucis digestifs et le mode de vie moderne :

Ainsi que l'a déclaré le docteur Miyamoto, l'acidité s'accumule dans l'estomac sous l'effet de l'ennui, mais il en va de même d'une surcharge d'activité, de cela j'ai une expérience intime ${ }^{39}$.

Sôseki ne cesse par ailleurs de multiplier ses critiques contre une " société aride, d'où la générosité est absente ${ }^{40}$ », « qui veut tout rendre rationnel ${ }^{41} »$, où le temps est morcelé et l'espace instable. Son faible appétit (vital) provient donc d'une difficulté fondamentale à évoluer dans un monde jugé inhabitable : " Pour faire un emprunt au langage bouddhique, je suis comme celui dont la maison est sans fin la proie des flammes ${ }^{42}$. »

\section{De la singularité du corps de l'autre}

Shiki, Sôseki : deux corps dans l'histoire, l'un et l'autre au croisement de l'appétit et de la souffrance, mais dans des configurations différentes.

Masaoka Shiki est un homme du désir (de manger), par-delà toutes les douleurs. La tuberculose vient sans doute restreindre drastiquement les conditions de déploiement de sa vitalité, mais sans y porter atteinte. Elle ne fait peut-être même que la renforcer et la magnifier.

Natsume Sôseki à l'inverse incarne, si l'on peut dire, la perte de l'appétit, les souffrances de l'inappétence. Chez lui, le désir ne se bat pas avec des limitations extérieures, il est corrompu à la racine. Cette impossibilité de s'emparer positivement du monde - des aliments, mais aussi des femmes - les conduit, lui et ses personnages, à une forme de débrayage par rapport à la réalité, retrait exprimé dans l'image du passage « de l'autre côté de la vitre ${ }^{43} »$. Pour Sôseki, la modernité, viciée en son essence, est synonyme de malaise et d'intranquillité.

38. Ibid., p. 33 ; Choses dont je me souviens, op. cit., p. 51.

39. Ibid., p. 16; Choses dont je me souviens, op. cit., p. 26.

40. Ibid., p. 78; Choses dont je me souviens, op. cit., p. 112.

41. Ibid., p. 59; Choses dont je me souviens, op. cit., p. 86.

42. Ibid., op. cit., p. 17; Choses dont je me souviens, op. cit., p. 31. Voir aussi Emmanuel Lozerand, «"En étrange pays dans mon pays lui-même." Le sentiment de l'exil intérieur chez quelques écrivains japonais modernes (Shiki, Ôgai, Sôseki) », dans Cécile Sakai et al. (dir.), Imaginaires de l'exil dans les littératures contemporaines en Chine et au Japon, Arles, Picquier, 2011 (à paraître). 43. Natsume Sôseki, Garasudo no naka, Tôkyô, Iwanami bunko, 1990, p. 9 ; trad. par René de Ceccatty et Nakamura Ryôji, À travers la vitre, Paris, Payot et Rivages, coll. " Rivages poche $», 2001$, p. 9 . 
Que nous apprennent ces expériences individuelles? Que ces deux corps, comme tous les autres, « japonais » ou non, sont singuliers, pluriels, complexes, conflictuels, historiques, fragiles. Cela implique-t-il, du coup, qu'il soit impossible de les caractériser? Pas nécessairement. Tout dépend de la nature et de la modalité des assemblages que l'on opère ${ }^{44}$. De même, par exemple, qu'il ne paraît plus acceptable de classer les corps en termes de races, de même ne semble-t-il pas nécessairement judicieux de les renvoyer en première instance à une improbable essence nationale, culturelle ou civilisationnelle ${ }^{45}$ (sans préjuger par ailleurs, le cas échéant, de l'existence éventuelle de caractéristiques partiellement communes, plus ou moins enracinées dans une histoire plus ou moins longue).

Même s'il ne fait aucun doute que la construction nationale japonaise moderne a marqué les corps qui ont croisé son chemin, ceux-ci ne sauraient pour autant se réduire à cette détermination. Car nul n'a les deux pieds dans son identité nationale. La question cruciale en vérité est donc celle des choix épistémologiques, de la détermination du point de vue selon lequel envisager le corps de l'autre. Veut-on considérer ce dernier « comme un insecte ${ }^{46}$ ", fixé sous le regard de l'anatomiste? Autrement dit du point de vue d'une histoire des vainqueurs ${ }^{47}$, des bien portants - que celle-ci soit commandée par le regard exotique des puissances occidentales (néo) coloniales ou par le narcissisme d'un orgueil chauvin?

Ou bien avons-nous à faire avec le corps de l'autre sous l'angle du désir, de la morale ou de la politique? En 1970, Roland Barthes critiquait vivement dans L'Empire des signes l'approche stéréotypique qui fait qu'un Français, « s'il voit un Japonais à Paris, le perçoit sous la pure abstraction de sa race (à supposer qu'il ne voie simplement en lui un Asiatique) ».

Après avoir unifié la race japonaise sous un seul type, il rapporte abusivement ce type à l'image culturelle qu'il a du Japonais, telle qu'il l'a construite à partir [...] de quelques photographies de presse, de quelques flashes d'actualité; et ce Japonais archétypique est assez lamentable : c'est un être menu, à lunettes, sans âge, au vêtement correct et terne, petit employé d'un pays grégaire.

Mais au Japon, explique Barthes de manière presque naïve, « tout change» :

44. Voir Daniel Colson, Petit lexique philosophique de l'anarchisme, Paris, Le Livre de Poche, 2001, articles « Corps », « Individuation », « Sujet » en particulier.

45. Pensons aux «slashs » de François Jullien qui à force de repasser sur le même (sur le corps, voir Le Nu impossible, Paris, Seuil, coll. « Points. Essais », 2005), malgré sa volonté de marquer des « écarts », finit par creuser des ornières, ce qui explique sans doute des propos sur le Japon, parfois condescendants, inexacts et convenus (Penser d'un dehors, entretiens avec Thierry Marchaisse, Paris, Seuil, 2000, p. 149-167).

46. Selon le mot de Cuvier cité par Stendhal dans ses Mémoires d'un touriste en 1838.

47. Sur la haine des vainqueurs, voir notre article, « Dans le temps, après la défaite. Sakaguchi Ango, Takeda Taijun et Takeuchi Yoshimi (1946-1948) », dans Michael Lucken, Anne BayardSakai et Emmanuel Lozerand (dir.), Le Japon après la guerre, Arles, Picquier, 2006, p. 109-125. 
La découverte est prodigieuse : les rues, les magasins, les bars, les cinémas, les trains déplient l'immense dictionnaire des visages et des silhouettes, où chaque corps (chaque mot) ne veut dire que lui-même et renvoie cependant à une classe; ainsi a-t-on à la fois la volupté d'une rencontre (avec la fragilité, la singularité) et l'illumination d'un type (le félin, le paysan, le rond comme une pomme rouge, le sauvage, le lapon, l'intellectuel, l'endormi, le lunaire, le rayonnant, le pensif), source d'une jubilation intellectuelle, puisque l'immaitrisable est maîtrisé ${ }^{48}$.

L'intérêt de l'approche barthésienne est d'individualiser les corps rencontrés au Japon, de les arracher à leur japonité, de redistribuer leurs régularités. Son inconvénient est de les priver, encore et toujours, de leur historicité. Ne serait-il pas possible a contrario de tisser des liens avec ces corps du/au Japon, de tirer des diagonales, de faire entendre des échos, qui les instituent en corps-frères, corps dialoguant, corps répondant, corps participant, comme les nôtres, de cette expérience singulière de l'historicité qui a pour nom modernité? On soulignerait alors une commune expérience de la pluralité, de la division, de la déstabilisation, prouvant ainsi en acte qu'il existe une forme d'universalité par le corps qui permet d'échapper aussi bien au relativisme culturaliste qu'à un universalisme transcendant ${ }^{49}$.

L'altérité des corps du/au Japon ne doit donc pas résider d'abord dans un ensemble de traits caractéristiques d'une masse inerte d' « ils ", mais plutôt dans la reconnaissance de ces autres comme de véritables autres, c'est-à-dire comme des sujets singuliers à en-visager, des « tu » en passe de devenir des $« \mathrm{je}^{50} »$, de nous parler, de nous entretenir. À leur tour ${ }^{51}$. Non sans opacité 52 , ni malentendu.

Emmanuel Lozerand Centre d'études japonaises (EA 1441) - Inalco (Paris)

48. Roland Barthes, L'Empire des signes, Paris, Flammarion, 1980, p. 127-129.

49. Voir Basile Doganis, La Pensée incarnée. Pratiques corporelles et arts gestuels japonais, Paris, Les Belles Lettres, 2012 (à paraître).

50. Irène Théry, « De la question du genre à celle de la personne. L'enjeu de l'interlocution », dans Philippe Corcuff, Christian Le Bart et François De Singly (dir.), L'Individu aujourd'hui. Débats sociologiques et contrepoints philosophiques, Rennes, Presses universitaires de Rennes, coll. « Res Publica », 2010, p. 378-381.

51. Sur le « tour de rôle », voir François Flahault, Le Sentiment d'exister, Paris, Descartes \& Cie, 2002, p. 412-413.

52. Maryse Condé, « Le monde à l'envers, ou l'empire des signes revisité », dans Michaël Ferrier (dir.), La Tentation de la France, la tentation du Japon. Regards croisés, Arles, Picquier, 2003, p. 186. 